\title{
Aplicación de la compresión lectora inferencial para el logro de las competencias en la resolución de problemas matemáticos con el método de Pólya en alumnos de la EPIME-UNTELS
}

\section{Application of inferential reading comprehension for the achievement of mathematical problem solving skills using Pólya's method in EPIME-UNTELS students}

\author{
DOI: $10.46932 / \operatorname{sjdv} 3 n 1-068$
}

Received in: Jan 30st, 2021

Accepted in: Feb 1th, 2022

\section{Dr. Roberto Pfuyo Muñoz}

Profesor en la Escuela profesional de Ingeniería Mecánica Eléctrica de la Universidad Nacional

Tecnológica de Lima Sur, Perú,

E-mail: rpfuyo@untels.edu.pe

\section{Magister Rosmel Iván Rodríguez Peceros}

Profesor en la Escuela profesional de Administración de Empresas de la Universidad Nacional

Tecnológica de Lima Sur, Perú

E-mail: rrodriguez@untels.edu.pe

\section{Magister Elizabeth Urrutia Huamán}

Profesora en la Escuela profesional de Contabilidad de la Universidad Tecnológica de los Andes, Perú

E-mail: ehuaman@utea.edu.pe

\section{Mtra. Maribel Osis Huamán}

Profesora en estudios generales de la Universidad Tecnológica de los Andes, Perú.

E-mail: mosis@utea.edu.pe

\section{RESUMEN}

La investigación surge a partir de la evidencia de los bajos niveles de desempeño de las capacidades en las asignaturas de matemática. Por lo tanto, la investigación pretende verificar comprensión lectora heurística y su interacción con el método de Pólya como destreza para fortalecer el logro de las competencias matemáticas en alumnos ingresantes. Si bien los alumnos leen el planteamiento del problema, debemos tener en cuenta que el alumno muestra problemas al examinar los datos, plantear un plan de solución y escoger el procedimiento de solución. Se implementó una estructura didáctica, organizada y secuencial con el objeto de abordar todos los pasos de este procedimiento. Esta investigación brindó a los alumnos la posibilidad de obtener herramientas para interpretar los problemas matemáticos, y de esta manera mejorar sus capacidades y promover a presentarse a nuevos desafíos sin las desconfianzas que ha creado el logro de las competencias matemáticas durante su formación profesional. El diseño de la investigación es descriptiva correlacional porque establece una relación entre los niveles de comprensión lectora inferencial y resolución de problemas matemáticos mediante la metodología de Pólya. De los resultados obtenidos, los niveles de comprensión inferencial y resolución de problemas matemáticos son significativas, se demostró que las etapas del método Pólya: Identificar el Problema, Elaborar el Plan, Implementar la Estrategia parar resolver el problema; contribuyen a estimular la 
comprensión lectora, motivan al estudiante a resolver problemas contextualizados con los contenidos de ingeniería y obligan al docente a utilizar técnicas didácticas, diferentes a la enseñanza de las matemáticas.

Palabras clave: Comprensión lectora heurística, Método Pólya, Competencias matemáticas, Formación profesional.

\begin{abstract}
The research arises from the evidence of the low levels of performance of skills in mathematics subjects. Therefore, the research aims to verify heuristic reading comprehension and its interaction with Pólya's method as a skill to strengthen the achievement of mathematical competences in incoming students. Although the students read the problem statement, we must take into account that the student shows problems when examining the data, formulating a solution plan and choosing the solution procedure. A didactic, organized and sequential structure was implemented in order to address all the steps of this procedure. This research provided the students with the possibility of obtaining tools to interpret mathematical problems, thus improving their abilities and promoting their ability to face new challenges without the mistrust created by the achievement of mathematical competences during their professional training. The research design is descriptive correlational because it establishes a relationship between the levels of inferential reading comprehension and mathematical problem solving using Pólya's methodology. From the results obtained, the levels of inferential comprehension and mathematical problem solving are significant, it was demonstrated that the stages of the Pólya method: Identify the Problem, Elaborate the Plan, Implement the Strategy to solve the problem; contribute to stimulate reading comprehension, motivate the student to solve problems contextualized with the engineering contents and oblige the teacher to use didactic techniques, different from the teaching of mathematics.
\end{abstract}

Keywords: Heuristic reading comprehension, Pólya Method, Mathematical competences, Professional training.

\title{
1 INTRODUCCIÓN
}

Las Escuelas profesionales de Ingeniería de las Universidades Públicas del Perú, se programan dentro del plan de estudios asignaturas de matemáticas, que es un elemento esencial para la mejora de los conocimientos de especialidad y aprendizaje en su formación como futuro Ingeniero, siendo la Matemática la asignatura un argumento importante dentro de los logros de las competencias básicas.

En consecuencia, el docente universitario cumple un papel muy importante en la etapa metodológica del aprendizaje de la matemática, puesto que es un pilar muy significativo para la formación de las competencias del proceso de aprendizaje, formulando un método y resolución de problemas de matemática.

Por tanto, la metodología y la didáctica en el proceso de enseñanza de la matemática se considera importante y necesario, por lo que la comprensión lectora inferencial es el procedimiento que permitirá habilitar resolver problemas de matemática con destrezas y habilidades.

En virtud de lo planteado, la influencia de la comprensión lectora inferencial y el método Pólya para resolver problemas de matemática demuestra su efectividad mediante los procedimientos de 
Comprensión, Planificación, Ejecución y Comprobación, y al considerar su fácil aplicación, por esta razón el uso de este método beneficia significativamente en la mejora del desempeño de las competencias de los alumnos de la Escuela Profesional de Ingeniería Mecánica Eléctrica (EPIME) de la Universidad Nacional Tecnológica de Lima Sur (UNTELS).

Es importante, precisar que es de gran utilidad la aplicación de la comprensión lectora inferencial y el método Pólya para la resolución de problemas en la enseñanza de la matemática, puesto que permitirá mejorar el desempeño académico, el cual ayudará a fortalecer el proceso de la enseñanza y aprendizaje simple (Pólya, 2009, p. 63).

\subsection{ANTECEDENTES}

La investigación de (Espinal \& Gelvez, 2019), tuvo como objetivo Aplicar el método de Pólya como estrategia para fortalecer la resolución de problemas matemáticos con operaciones básicas en estudiantes de los grados tercero y cuarto de primaria del Colegio Municipal Aeropuerto, mediante la implementación de una guía didáctica básica basada en el método de George Pólya como estrategia pedagógica, puesto que constituye un método planteada con el objetivo de facilitar el aprendizaje de los alumnos, el diseño de la investigación fue cualitativo de acción. La conclusión que resulto que al analizar los resultados de la prueba de evaluación diagnóstica se verificó que la mayoría presentaba dificultad para leer y comprender problemas matemáticos e identificar la operación requerida para encontrar la solución, lo que origina el bajo rendimiento en el área, los estudiantes logran leer el enunciado de un problema, pero se debe tener en cuenta que presentan dificultades al analizar los datos, proponer una estrategia de solución y elegir el método de solución indicado. La investigación brindó a los estudiantes la posibilidad de adquirir herramientas para interpretar los problemas matemáticos, mejorar sus competencias y motivarlos a enfrentarse a nuevos retos sin los temores que ha generado el área de matemáticas.

(Cuello y otros, 2021), desarrollo el trabajo de investigación "Método de Pólya: una alternativa en la resolución de problemas matemáticos" cuyo objetivo fue la aplicación de la Metodología de George Pólya en el mejoramiento del desempeño académico de los estudiantes de grado noveno, de educación básica secundaria de dos Instituciones Educativas del Distrito de Barranquilla. La investigación tuvo resultados en los niveles de comprensión y de desempeño, y demostraron que las etapas de la metodología de Pólya: Identificar el Problema, Elaborar el Plan, Implementar la Estrategia parar resolver el problema y hacer una Visión Retrospectiva del mismo; contribuyen a estimular la comprensión lectora, motivan al estudiante a resolver problemas contextualizados y obligan al docente a utilizar técnicas didácticas, diferentes a la operacionalización de las matemáticas. 
(Díaz Olmos \& Rodríguez Rayo, 2021), en el trabajo denominado "Discurso docente desde la metodología de Pólya en la resolución de problemas matemáticos”, desarrolla un análisis, que las instituciones educativas el docente se convierte en un personaje más importante del sistema educativo. Las asignaturas de matemáticas, deberá contener las metodologías que motive al estudiante a comprender, plantear y resolver problemas matemáticos. De acuerdo al Índice Sintético de la Calidad Educativa -ISCE existe un bajo desempeño en la competencia de resolución de problemas en la I.E.D Tercera Mixta en el nivel de básica. De acuerdo a ello, realizaron la investigación con el objetivo de comprender desde el discurso docente y la metodología de Pólya sus aportes a la resolución de problemas matemáticos. La metodología se basa en un enfoque cualitativo y un paradigma crítico social, las técnicas son revisión documental, entrevista semiestructurada, grupo focal y grupo de discusión. Los resultados arrojan que en las guías de trabajo y las metodologías pedagógicas no mantienen una unificación de criterios. En definitiva, el aprendizaje es afectado al no definirse una relación lógica entre las partes.

\subsection{OBJETIVO}

El proceso de enseñanza de aprendizaje significativa de la matemática, exigen a los docentes implementar estrategias metodológicas que faciliten la resolución de problemas matemáticos y a los estudiantes el procedimiento de resolución de problemas matemática mediante la comprensión lectora inferencial a través el procedimiento y estrategias de Pólya, los cuales deben estar relacionadas a experiencias cotidianas, es decir, el procedimiento de resolución será significativo si los problemas matemáticos son extraídos de las realidades de aplicaciones en ingeniería mecánica eléctrica. Por tanto, el objetivo es determinar la relación de los niveles de comprensión lectora inferencial con la resolución de problemas matemáticos. Es así que los resultados de aplicar la metodología de Pólya, demuestra la hipótesis de esta investigación, cuyo estudio cuantitativo tuvo lugar en los estudiantes ingresantes a la Escuela Profesional de Ingeniería Mecánica Eléctrica de la UNTELS.

\section{METODOLOGÍA}

\subsection{TIPO DE INVESTIGACIÓN}

El tipo de investigación es descriptivo correlacional. Descriptivo porque verifica las particularidades más importantes de los indicadores de las variables y dimensiones. (Hernández Sampieri et al., 2014, p. 102). Es correlacional porque se determina la relación de las variables intervinientes, comprensión lectora inferencial y el logro de competencias de resolución de problemas matemáticos mediante el método de Pólya, que sostienen que los estudios de correlación miden dos o más variables para analizar las correlaciones y ver si están relacionadas entre sí. 


\subsection{NIVEL DE INVESTIGACIÓN}

El nivel de investigación es descriptivo y causal ya que tiene como objetivo abordar los orígenes de los eventos físicos o sociales. (...) la importancia radica en saber por qué está sucediendo este fenómeno. (...) y es más estructurado e investigado que en otros niveles, (Charaja, 2018, p. 44).

\subsection{MÉTODO DE INVESTIGACIÓN}

El método aplicado es cuantitativo, según Kerlinger citado por (Vásquez Soto \& Moya Amador, 2018) es de tipo no experimental, descriptivo correlacional porque se pretende manifestar o explicar la relación significativa de las variables mediante el uso de los coeficientes de correlación de Pearson, en la población de análisis de los estudiantes de la EPIME.

\subsection{POBLACIÓN Y MUESTRA}

La población está constituida por 46 estudiantes del primer semestre de la EPIME durante el semestre académico del 2021-I de la UNTELS. La muestra es igual a la población y se procesó con 46 alumnos, se utilizó el muestreo aleatorio simple por conveniencia.

\subsection{TÉCNICAS E INSTRUMENTOS DE RECOLECCIÓN DE DATOS}

El instrumento utilizado se efectuó mediante un examen con pruebas virtuales auto administradas, como técnicas cuantitativas que permitieron recolectar información proveniente de los estudiantes de la EPIME que son parte de la muestra, de comprensión lectora inferencial y la resolución de problemas matemáticos mediante las cuatro etapas del método de Pólya. Por tanto, la respuesta correcta corresponde a cada de la respuesta correcta, anulándose las demás. La encuesta considerada fue sobre los niveles de comprensión lectora inferencial y resolución de problemas matemáticos en sus cuatro etapas. (Vicente \& López, 2016).

\subsection{COMPRENSIÓN LECTORA NIVEL INFERENCIAL}

Considera desarrollar las ideas y la información planteada en el texto. Donde las inferencias se reflejan en el estudiante al usar ideas del texto y la experiencia personal. Los reactivos de inferencia utilizados son: Inferencia de detalles, principal, secuencias, comparaciones, relaciones de causa, rasgos de carácter. Este elemento permitió distinguir un hecho y un juicio frente a un comportamiento. 


\subsection{RESOLUCIÓN DE PROBLEMAS MATEMÁTICOS: MÉTODO DE PÓLYA}

\section{Etapa 1: Comprensión del problema}

Este paso es inevitable, donde es imposible resolver el problema cuya declaración no se entiende. Sin embargo, en la mayoría de los casos, los estudiantes realizaron sus operaciones y aplicaron fórmulas sin pensar dos veces en lo que se les pide. Lo cual revela una total incomprensión de lo que es el problema y plantea una situación muy difícil para el profesor, vicios arraigados del pensamiento, quizás adquiridos durante muchos años.

\section{Etapa 2: Planificación}

Esta etapa es la más delicada porque no solo está relacionada con el conocimiento en la etapa de razonamiento, sino también con la imaginación y la creatividad, que dio como resultado un esquema y otras representaciones. Se observo que las preguntas que Pólya relaciona en esta etapa pretenden llevar el problema al plano de solución.

\section{Etapa 3: Ejecución del plan}

En este paso, se revisó la verificación de cada uno de los pasos del plan para verificar si estaban correctos. Si el plan está bien diseñado, su realización es viable y el estudiante tiene los conocimientos y la formación necesarios, y pudo llevarlo a cabo sin problemas. Sin embargo, en general, se han encontrado dificultades en esta etapa que nos obligó a volver a la etapa anterior para hacer ajustes al plan o incluso modificarlo por completo.

\section{Etapa 4: Comprobar el resultado}

Se insistió el método de Pólya por su importancia, no solo porque verifico las medidas tomadas y verificar su exactitud, sino porque la retrospectiva nos llevó a resultados que generalizaron, aumentaron y reforzaron para que se pueda encontrar la solución. Por lo tanto, las incógnitas se han identificado y tenido en cuenta durante este paso.

\subsection{VALIDES DE LA ESTRUCTURA INTERNA}

La validez del constructo se efectuó mediante el análisis de su estructura interna por medio de un análisis factorial exploratorio, para ello, se aplicó es estadístico descriptivo KMO (Kaiser-Meyer-Olkin) cuyo resultado obtenido debe ser superior a cero. Se analizo utilizando una muestra estratificada piloto de 46 jueces con características similares de la población llevado a cabo en el estudio. Los resultados de validez interna por dimensión se presentan en el Tabla 1 . 
Tabla 1. Validez de Estructura Interna KMO de las Áreas Comprensión Lectora Inferencial y Método de Pólya

\begin{tabular}{|l|c|c|c|c|c|c|}
\hline $\begin{array}{l}\text { KMO y prueba de } \\
\text { Bartlett }\end{array}$ & $\begin{array}{c}\text { Compresión } \\
\text { lectora: nivel } \\
\text { inferencial }\end{array}$ & $\begin{array}{c}\text { Comprensió } \\
\text { n del } \\
\text { problema }\end{array}$ & $\begin{array}{c}\text { Planificació } \\
\mathrm{n}\end{array}$ & $\begin{array}{c}\text { Ejecución } \\
\text { del plan }\end{array}$ & $\begin{array}{c}\text { Comprobar } \\
\text { resultado }\end{array}$ \\
\hline $\begin{array}{l}\text { Medida de adecuación } \\
\text { muestral de Kaiser- } \\
\text { Meyer-Olkin. }\end{array}$ & 0.742 & 0.693 & 0.685 & 0.763 & 0.801 \\
\hline $\begin{array}{l}\text { Prueba } \\
\text { de } \\
\text { esfericid } \\
\text { ad de } \\
\text { Bartlett }\end{array}$ & $\begin{array}{l}\text { Chi-cuadrado } \\
\text { aproximado }\end{array}$ & 14029.08 & 13554.8 & 11475.2 & 14537.4 & 1120.94 \\
\cline { 2 - 7 } & Sig. & 0.000 & 0.000 & 0.000 & 0.000 & 3 \\
\hline
\end{tabular}

De esta manera podemos concluir que la muestra piloto coincide con los resultados de la prueba de control aplicado. Por lo tanto, existe una correlación entre los elementos de las áreas y está relacionado con una medición única de los ítems del cuestionario. En otras palabras, mide lo que quieres medir.

\section{ANÁLISIS DE CONFIABILIDAD}

La validación de los instrumentos se analizó con una muestra piloto de 46 jueces de características similares de la población, para la determinación de la confiabilidad del instrumento se utilizó el coeficiente alfa de Cronbach. El resultado de confiabilidad del instrumento según Alfa de Cronbach es superior a 0,5 en cada dimensión y muestra valores significativos dentro del rango aplicable y confiable, por lo que, mide lo que pretende medir.

Tabla 2. Nivel de Confiabilidad Áreas: Compresión Lectora Inferencial y Resolución de Problemas Matemáticos por el Método de Pólya

\begin{tabular}{|l|l|c|c|}
\hline Ítem & Dimensiones & $\begin{array}{c}\text { Alfa de } \\
\text { Cronbach }\end{array}$ & $\begin{array}{c}\mathbf{N}^{\circ} \text { de } \\
\text { elementos }\end{array}$ \\
\hline 1 & Comprensión lectora inferencial & 0,832 & 9 \\
\hline 2 & Comprensión del problema & 0,678 & 2 \\
\hline 3 & Planificación & 0,721 & 2 \\
\hline 4 & Ejecución del plan & 0,692 & 2 \\
\hline 5 & Comprobar resultado & 0,744 & 2 \\
\hline
\end{tabular}

\section{RESULTADOS}

La realización de esta investigación requirió la aplicación de un instrumento para recolectar información de los sujetos de estudio que pertenecen a la muestra de investigación. Para ello, se utilizó la encuesta como técnica el cuestionario como instrumento porque su formulación se basó en preguntas escritas.

Las preguntas de los ítems de ambas variables tuvieron la siguiente valoración: Malo, Regular, Bueno, y Muy bueno. Las evaluaciones consistieron en la adecuada formulación de preguntas verbales o de desempeño, con el objetivo de descubrir para diagnosticar el desempeño académico, en particular cognitivo, en su aspecto específico o total. Las pruebas escritas, fueron tipo objetivas, por su forma de 
responderlas porque intentan eliminar, en lo posible, la subjetividad del docente a la hora de analizar, procesar y evaluar la prueba.(Gottheil et al., s. f.)

Compresión lectora inferencial y la resolución de problemas matemáticos mediante el método de Pólya

Tabla 3. Relación del Compresión lector inferencial y Resolución de Problemas Matemáticos: Etapas de Planificación y Ejecución del plan

\begin{tabular}{|c|c|c|c|c|c|c|c|c|c|c|c|}
\hline & \multicolumn{10}{|c|}{$\begin{array}{l}\text { Resolución de Problemas Matemáticos: Dimensión Planificación y } \\
\text { Ejecución del Plan }\end{array}$} \\
\hline & & \multicolumn{2}{|c|}{ Malo } & \multicolumn{2}{|c|}{ Regular } & \multicolumn{2}{|c|}{ Bueno } & \multicolumn{2}{|c|}{ Muy bueno } & \multicolumn{2}{|c|}{ Total } \\
\hline & & & $\%$ & $\mathrm{f}$ & $\%$ & $\mathrm{f}$ & $\%$ & $\mathrm{f}$ & $\%$ & f & $\%$ \\
\hline \multirow{5}{*}{$\begin{array}{l}\text { Compresión } \\
\text { lectora a } \\
\text { nivel } \\
\text { inferencial }\end{array}$} & & 4 & $8.7 \%$ & 4 & $8.7 \%$ & 1 & $2.2 \%$ & 1 & $2.2 \%$ & 10 & $21.7 \%$ \\
\hline & Regular & 2 & $4.3 \%$ & 4 & $8.7 \%$ & 3 & $6.5 \%$ & 2 & $4.3 \%$ & 11 & $23.9 \%$ \\
\hline & Bueno & 2 & $4.3 \%$ & 1 & $2.2 \%$ & 2 & $4.3 \%$ & 7 & $15.2 \%$ & 12 & $26.1 \%$ \\
\hline & Muy Bueno & 1 & $2.2 \%$ & 3 & $6.5 \%$ & 4 & $8.7 \%$ & 5 & $10.9 \%$ & 13 & $28.3 \%$ \\
\hline & Total & 9 & $19.6 \%$ & 12 & $26.1 \%$ & 10 & $21.7 \%$ & 15 & $32.6 \%$ & 46 & $100.0 \%$ \\
\hline
\end{tabular}

Figura 1. Relación del comprensión lectora inferencial y Resolución de Problemas (Etapa de Planificación y Ejecución del Plan)

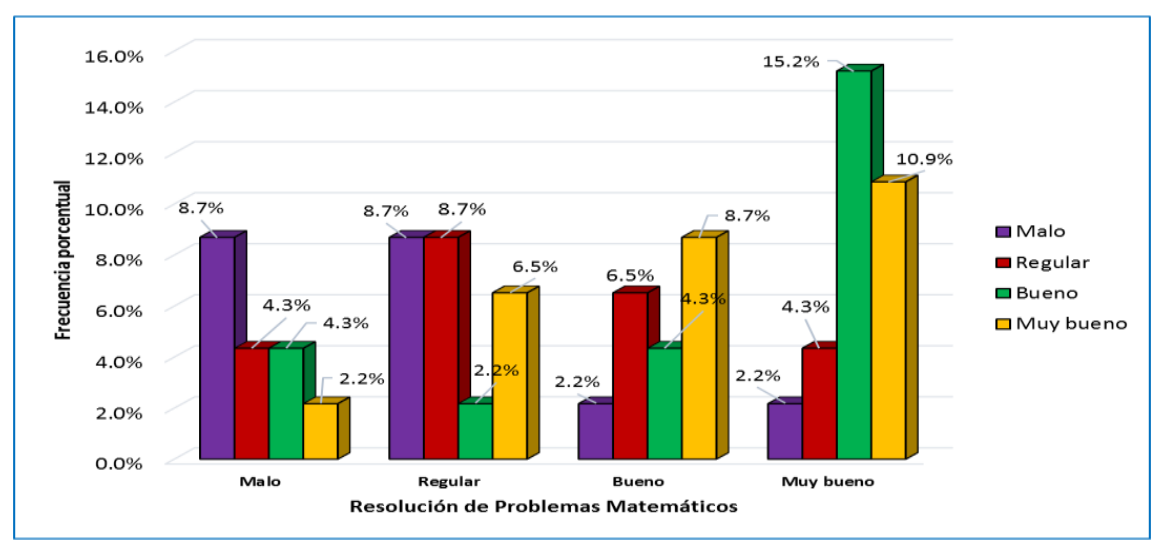

En la Figura 1 se aprecia que el 15.20\% de los alumnos se encuentran emplazados en la escala de bueno dentro del nivel inferencial de compresión lectora están ubicados en el nivel muy bueno en la resolución de problemas matemáticos, seguido de $10.9 \%$ de estudiantes que se encuentran ubicados en la escala de muy bueno y muy bueno en la resolución de problemas matemáticos. En conclusión, los alumnos que se encuentran ubicados en la escala bueno en el nivel de comprensión lectora inferencial están ubicados en el nivel de muy bueno en resolución de problemas matemáticos.

\subsection{PRUEBA DE HIPÓTESIS}

La validación de la hipótesis la compresión lectora inferencial y la resolución de problemas matemáticos con las etapas Planificación y ejecución del plan del método de Pólya de los alumnos EPIME, para lo cual se ha validado la hipótesis. 
Tabla 4. Correlación de la comprensión lectora inferencial y la resolución de problemas matemáticos - Método Pólya. Etapa Planificación y Ejecución del Plan

\begin{tabular}{|c|c|c|c|c|}
\hline \multicolumn{5}{|c|}{ Correlaciones } \\
\hline & & & $\begin{array}{l}\text { Resolución de } \\
\text { Problemas } \\
\text { Matemáticos } \\
\text { (Planificación y } \\
\text { ejecución del } \\
\text { plan) } \\
\end{array}$ & $\begin{array}{c}\text { Nivel } \\
\text { Inferencial }\end{array}$ \\
\hline \multirow[t]{2}{*}{$\begin{array}{l}\text { Rho de } \\
\text { Spearman }\end{array}$} & $\begin{array}{l}\text { Resolución de } \\
\text { Problemas } \\
\text { Matemáticos }\end{array}$ & $\begin{array}{l}\text { Coeficiente de } \\
\text { correlación } \\
\text { Sig. (bilateral) } \\
\mathrm{N} \\
\end{array}$ & $\begin{array}{r}1,000 \\
46 \\
\end{array}$ & $\begin{array}{r}, 801^{*+} \\
, 000 \\
46\end{array}$ \\
\hline & Nivel Inferencial & $\begin{array}{l}\text { Coeficiente de } \\
\text { correlación } \\
\text { Sig. (bilateral) } \\
\text { N }\end{array}$ & $\begin{array}{r}, 801^{4 *} \\
, 000 \\
46\end{array}$ & $\begin{array}{r}1,000 \\
46\end{array}$ \\
\hline
\end{tabular}

Como se aprecia en el Tabla 4, el valor significancia es de 0,000 , valor inferior a 0,05 , por lo tanto, se rechaza la hipótesis nula, y se puede afirmar que, con un nivel de confianza del 95\% existe una relación significativa entre los niveles de comprensión lectora inferencial y la resolución de problemas matemáticos mediante el método de Pólya de los alumnos de la EPIME-UNTELS, Asimismo, es necesario destacar que la correlación es de $0,801^{* *}$ que establece un alto nivel de correlación muy cercano a 1 . Comprensión Lectora Inferencial y la Resolución de Problemas Matemáticos "Método Pólya” a Nivel

\section{General}

Tabla 5. Relación del Compresión Lectora Inferencial y Resolución de Problemas Matemáticos a Nivel General

\begin{tabular}{|c|c|c|c|c|c|c|c|c|c|c|c|}
\hline & \multicolumn{10}{|c|}{$\begin{array}{c}\text { Resolución de Problemas Matemáticos Método del Pólya: Nivel } \\
\text { General }\end{array}$} \\
\hline & & \multicolumn{2}{|c|}{ Malo } & \multicolumn{2}{|c|}{ Regular } & \multicolumn{2}{|c|}{ Bueno } & \multicolumn{2}{|c|}{ Muy bueno } & \multicolumn{2}{|c|}{ Total } \\
\hline & & f & $\%$ & f & $\%$ & f & $\%$ & f & $\%$ & f & $\%$ \\
\hline \multirow{5}{*}{$\begin{array}{l}\text { Compresión } \\
\text { lectora a } \\
\text { nivel } \\
\text { inferencial }\end{array}$} & Malo & 1 & $2.2 \%$ & 1 & $2.2 \%$ & 1 & $2.2 \%$ & 1 & $2.2 \%$ & 4 & $8.7 \%$ \\
\hline & Regular & 3 & $6.5 \%$ & 8 & $17.4 \%$ & 2 & $4.3 \%$ & 2 & $4.3 \%$ & 15 & $32.6 \%$ \\
\hline & Bueno & 4 & $8.7 \%$ & 2 & $4.3 \%$ & 5 & $10.9 \%$ & 3 & $6.5 \%$ & 14 & $30.4 \%$ \\
\hline & Muy Bueno & 1 & $2.2 \%$ & 3 & $6.5 \%$ & 2 & $4.3 \%$ & 7 & $15.2 \%$ & 13 & $28.3 \%$ \\
\hline & Total & 9 & $19.6 \%$ & 14 & $30.4 \%$ & 10 & $21.7 \%$ & 13 & $28.3 \%$ & 46 & $100.0 \%$ \\
\hline
\end{tabular}

Figura 2. Relación Comprensión Lectora Inferencial y Resolución de Problemas - Método Pólya a nivel General

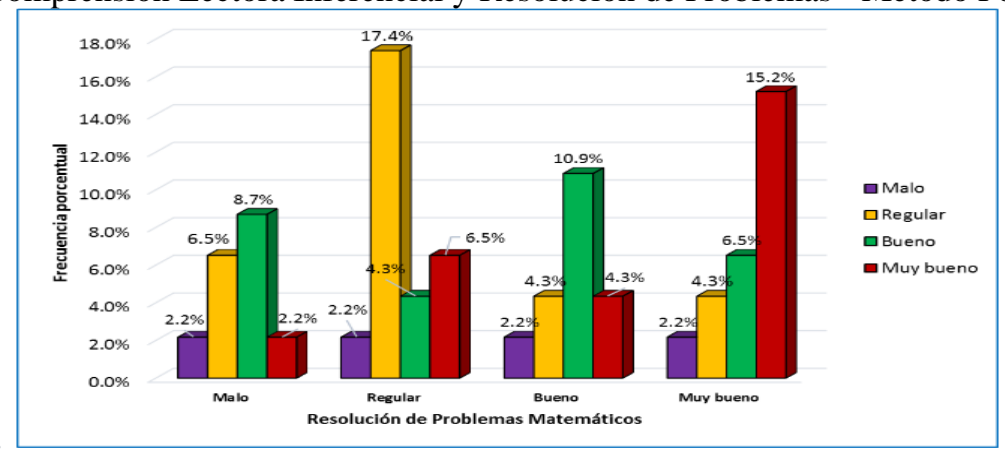


De acuerdo al Tabla 5 y la Figura 2 se aprecia que el 15.20\% de alumnos se encuentran ubicados en la escala muy bueno y $10.90 \%$ en la escala bueno, dentro de los niveles de comprensión lectora están ubicados en el nivel muy bueno en la resolución de problemas matemáticos. En conclusión; los estudiantes que se encuentran ubicados en la escala muy bueno en el nivel de comprensión lectora inferencial se encuentran ubicados en el nivel de muy bueno en la resolución de problemas matemáticos, es decir, a mejor comprensión lectora inferencial están en un nivel de muy bueno en resolución de problema matemáticos.

\subsection{PRUEBA DE HIPÓTESIS}

Se ha validado la hipótesis de compresión lectora inferencial y la resolución de problemas matemáticos con la dimensión Planificación y ejecución del plan de método de Pólya de los alumnos EPIME, para lo cual se ha validado la hipótesis.

Tabla 6. Correlación ente Compresión Lectora Inferencial y Resolución de Problemas Matemáticos- Método Pólya Correlaciones

\begin{tabular}{|c|c|c|c|}
\hline & & $\begin{array}{l}\text { Resolución de } \\
\text { Problemas } \\
\text { Matemáticos }\end{array}$ & $\begin{array}{c}\text { Niveles de } \\
\text { Comprensión } \\
\text { Lectora } \\
\text { Inferencial }\end{array}$ \\
\hline $\begin{array}{l}\text { Resolución de Problemas } \\
\text { Matemáticos }\end{array}$ & $\begin{array}{l}\text { Correlación de Pearson } \\
\text { Sig. (bilateral) } \\
\mathrm{N}\end{array}$ & $\begin{array}{r}1 \\
46 \\
\end{array}$ & $\begin{array}{r}, 751^{* *} \\
, 000 \\
46 \\
\end{array}$ \\
\hline $\begin{array}{l}\text { Niveles de Comprensión } \\
\text { Lectora Inferencial }\end{array}$ & $\begin{array}{l}\text { Correlación de Pearson } \\
\text { Sig. (bilateral) } \\
\mathrm{N}\end{array}$ & $\begin{array}{r}, 751^{* *} \\
, 000 \\
46\end{array}$ & $\begin{array}{r}1 \\
46\end{array}$ \\
\hline
\end{tabular}

De acuerdo a los resultados en el Tabla 6, el valor significancia es de 0,000, inferior a 0,05, por tanto, se rechaza hipótesis nula, y se puede confirmar con un nivel de confianza del 95\%, existe correlación significativa entre los niveles de comprensión lectora inferencial y la resolución de problemas matemáticos mediante el método de Pólya de los alumnos de la EPIME-UNTELS. Por lo tanto, de acuerdo al coeficiente de Pearson existe una correlación de $0,751^{* *}$, que representa una correlación positiva alta.

\section{CONCLUSIONES}

El resultado de la investigación demuestra que existe relación directa y significativa entre los niveles de comprensión lectora inferencial y la resolución de problemas matemáticos con el método de Pólya en los Alumnos de la EPIME. Asimismo, es indispensable destacar que la correlación de 0,751 muestra el valor de correlación que existe entre las variables intervinientes. Por tanto, de acuerdo al 
resultado del coeficiente de Pearson se observa una correlación de 0,751 , lo que representa una correlación positiva alta, lo que establece un alto nivel de correlación muy cercano a 1. La ausencia del nivel ejecución del plan e interpretación de los resultados fue quizás inesperado el haber encontrado que el nivel de correlación es medio. Por lo tanto, es importante señalar que los alumnos que tienen un buen y excelente el análisis inferencial de la comprensión lectora tienen facilidad en la resolución de problemas matemáticos, de acuerdo a las cuatro etapas de resolución de problemas matemáticos, Planificación, Ejecución del plan y Comprobar los resultados.

Mediante la implementación de la metodología, se verifica que la metodología de Pólya para la resolución de problemas matemáticos, es una estrategia de enseñanza significativa que mejora la comprensión lectora inferencial y, por lo tanto, el desempeño académico del docente. La implementación de las estrategias didácticas contextualizadas en ingeniería, estimulan al estudiante el pensamiento crítico, lógico y matemático, exigiendo al docente la innovación e implementación de estrategias para el proceso de enseñanza aprendizaje, consiguientemente, las matemáticas cambian su aspecto tradicional y se vuelven motivadoras en el estudiante.

\section{LIMITACIONES}

En cuanto a las limitaciones en el trabajo, se puede precisar que es viable la aplicación con poblaciones similares al objeto de estudio, toda vez que se ha efectuado la validez y confiabilidad de los cuestionarios y pruebas de pretest y postest, instrumentos que fueron adecuados para la aplicación a estudiantes de educación superior universitaria y tecnológica. En cuanto a las limitaciones de tiempo y recursos se tuvo dificultades, puesto que los estudiantes no se encontraron virtualmente en el momento de la aplicación de las pruebas y cuestionarios, sin embargo, estos no afectaron significativa en el procedimiento del estudio.

\section{RECOMENDACIONES}

Los investigadores interesados en continuar nuestra investigación podrían tener en consideración los resultados de la investigación ya que después de haber comprobado la correlación significativa que existe entre las variables, por lo que, los funcionarios de la UNTELS, deben programar capacitación de inducción docente, en los niveles de comprensión de lectura como la resolución de problemas matemáticos mediante el método de Pólya de esta forma mejorar los aprendizajes y el rendimiento académico de los alumnos, reiterando que la comprensión lectora inferencial ejerce influencia significativa en la resolución de problemas matemáticos. Se recomienda que existe temas por explorarse en lo que se refiere al uso de la metodología de Pólya para la resolución de problemas matemáticos. 


\section{REFERENCIAS}

Charaja, F. (2018). El MAPIC en la Metodología de la Investigación. EDITORIAL: Sagitario Impresores Puno Perú.

Cuello, D., Valera, L., \& Barrios, A. (2021). Método de Pólya: una alternativa en la resolución de problemas matemáticos. Ciencias e Ingeniería, 8(2). https://doi.org/5716273

Díaz Olmos, K., \& Rodríguez Rayo, C. (2021). Discurso docente desde la metodología de Pólya en la resolución de problemas matemáticos. Corporación Universidad de la Costa, 1(2), 2-188.

Espinal, M., \& Gelvez, D. (2019). Método de Pólya como estrategia pedagógica para fortalecer la competencia resolución de problemas matemáticos con operaciones básicas. Zona Próxima (31), 8-25.

Gottheil, B., Brenlla, M. E., Barreyro, J. P., Pueyrredón, D., Aldrey, A., Buonsanti, L., Freire, L., \& Rossi, A. I. (s. f.). Eficacia del programa "Lee Comprensivamente" para la enseñanza de estrategias de comprensión lectora en estudiantes de Educación Primaria. 13.

Hernández Sampieri, R., Fernández Collado, C., \& Pilar Baptista Lucio, M. (2014). Metodología de la investigación. McGraw-Hill.

Pólya, G. (2009). Mathematical discovery: On understanding, learning, and teaching problem solving. Ishi Press.

Vásquez Soto, E., \& Moya Amador, A. M. (2018). Factores que influyen en el proceso de comprensión lectora y la resolución de problemas matemáticos según el Modelo Pólya en los estudiantes de grado tercero de las instituciones educativas Buenos Aires y Ricaurte del municipio de Soacha. instname:Universidad de los Andes. https://repositorio.uniandes.edu.co/handle/1992/34429

Vicente, J. S. Y., \& López, J. L. R. (2016). Aplicación del método PÓLYA para mejorar el rendimiento académico de matemática en los estudiantes de secundaria. INNOVA Research Journal, 1(10), 12-20. https://doi.org/10.33890/innova.v1.n10.2016.53

Yangali Vicente, J. S., \& Rodríguez López, J. L. (2016). Aplicación del Método Pólya para mejorar el Rendimiento Académico de Matemática en los Estudiantes de Secundaria. Innova Research Journal, 1(10), 12-20. https://doi.org/10.33890/innova.v1.n10.2016.53 